\title{
Wilhelm Conrad Röntgen - der Mann hinter der Entdeckung der „X-Strahlen“
}

\section{Anna-Katharina Kätker, Deutsches Röntgen-Museum}

Die bergische Stadt Lennep, heute ein Stadtteil von Remscheid, war seit dem frühen 18. Jahrhundert ein Zentrum des Textilgewerbes. Als Wilhelm Conrad Röntgen dort am 27. März 1845 auf die Welt kam, blickte seine Familie schon auf eine rund 120 Jahre währende Tätigkeit im Tuchhandel zurück. Nichts ließ zunächst in seiner frühen Jugend darauf schließen, dass er dieser Familientradition nicht folgen würde. Sein Vater war Kaufmann und Tuchfabrikant und auch seine Mutter entstammte einer alten Lenneper Kaufmannsfamilie, die jedoch in den Niederlanden ansässig war. Die familiären Beziehungen zu den Niederlanden und vermutlich auch die Auswirkungen der 1848er-Revolution mit den einhergehenden wirtschaftlichen Einbußen ließen Vater, Mutter und Sohn, denn Geschwister hatte der kleine Wilhelm nicht, nach Apeldoorn übersiedeln. So verließ Röntgen schon mit 3 Jahren seine Geburtsstadt, die er nur ein einziges Mal, und zwar auf einer Durchreise im Jahre 1911, wiedersehen sollte. Ob es eine sentimentale Verbundenheit mit dem Ort seiner Geburt war oder die Freude über die ihm 1896 erwiesene Ehrenbürgerwürde der Stadt Lennep - er hinterließ ihr in seinem Nachlass 30000 Mark.

Der kleine Wilhelm wurde zunächst auf eine Privatschule in Apeldoorn geschickt und verlebte wohl eine glückliche Kindheit in den Niederlanden, die ihn stark prägte. Noch in späteren Jahren, als er schon wieder längere Zeit in der Schweiz und in Deutschland gelebt hatte, wird über ihn berichtet: „Seine Conversationssprache ist nicht immer ohne einen fremden Anklang, der Holländer verläugnet (!) sich nicht völlig. “ Mit 17 Jahren, im Jahr 1862, begann er die Technische Schule in Utrecht zu besuchen und wohnte im Haus des bekannten Chemieprofessors Jan Willem Gunning, der seine Faszination für die Naturwissenschaften stärkte. Die Technische Schule sollte als Vorbereitung auf das Studium eines technischen Berufs dienen, und so war zu diesem Zeitpunkt eventuell noch geplant, dass der junge Röntgen der Familientra-

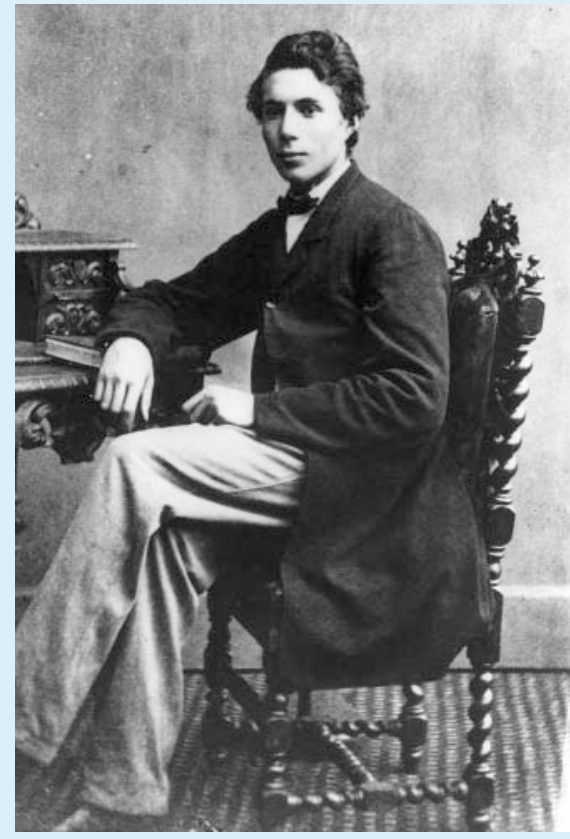

Röntgen als Schüler in Utrecht. (c) Deutsches Röntgenmuseum.

dition folgen und wie sein Vater Tuchfabrikant würde. Doch es kam anders. Denn 1863 flog Röntgen von der Schule - wer hätte das von einem zukünftigen Nobelpreisträger gedacht?! Nach Röntgens eigener Aussage wurde er der Schule verwiesen, da er den Namen eines Schülers nicht verraten wollte, der Urheber eine Lehrerkarikatur war. Fortan trieb Röntgen Privatstudien und war als Gasthörer an der Universität Utrecht eingeschrieben. Hier berichtete ihm ein Kommilitone, dass das Eidgenössische Polytechnikum in Zürich Studenten auch ohne Abitur annehme und Röntgen zögerte nicht lange. 1865 siedelte er nach Zürich um und schrieb sich für Maschinenbau ein.

Die Studienzeit in der Schweiz wird für Röntgens Berufs- wie auch Privatleben eine entscheidende Rolle spielen. Hier machte er die Bekanntschaft des jungen Physikprofessors August Kundt, an den sich Röntgen später wie folgt erinnert: „der mich in die Physik einführte und mich aus der Unsicherheit über meine Zukunft herausriß“. Kundt ermöglichte Röntgen darüber hinaus nach seinem erfolgreich abgeschlossenen Ma-

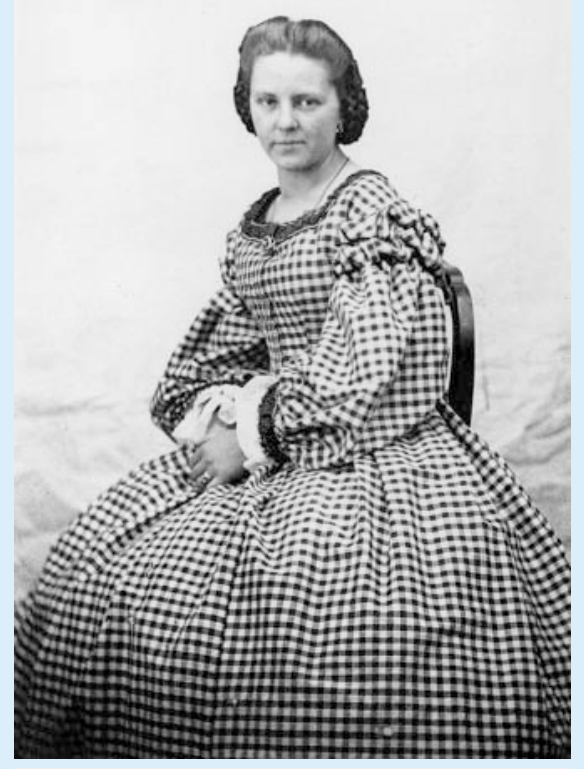

Die junge Anna Bertha Ludwig um 1870. (C) Deutsches Röntgenmuseum.

schinenbau-Studium die Promotion im Fachbereich Physik. 1869 erlangte Röntgen mit seiner Dissertation „Studien über Gase“ die Doktorwürde und einer wissenschaftlichen Karriere schien zunächst nichts im Wege zu stehen. Er verbrachte seine Zeit aber nicht ausschließlich mit Studien und Forschung, sondern war auch regelmäßiger Besucher in der Gaststätte „Zum Grünen Glas“. Einerseits wegen der feucht-fröhlichen Stimmung, die im Gasthaus herrschte, andererseits aber auch weil er sich zu einer der Wirtstöchter im Besonderen hingezogen fühlte. 1872 heiratete er schließlich die 6 Jahre ältere Anna Bertha Ludwig. Auch wenn die Ehe nicht im Sinne von Röntgens Eltern war, denn sie hätten sich eine Frau höheren Standes für ihren Sohn gewünscht, verlief sie bis zum Tode Anna Berthas im Jahr 1919 ausgesprochen glücklich. Röntgen pflegte seine Frau in den letzten Wochen ihrer Krankheit hingebungsvoll. Die Ehe blieb zum Leidwesen beider kinderlos, jedoch nahmen sie 1887 Berthas 6-jährige Nichte Josephine zu sich und adoptierten sie 15 Jahre später. 


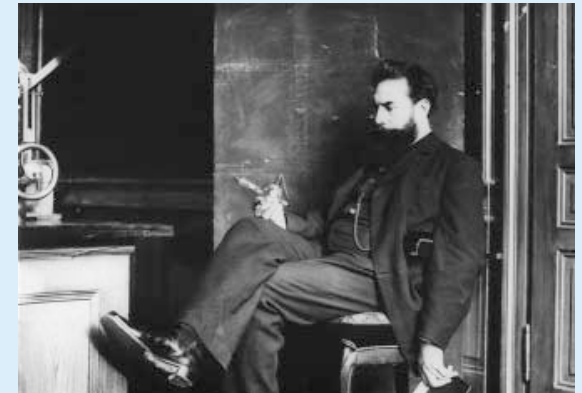

Röntgen mit Röntgenröhre im Würzburger Labor. @ Deutsches Röntgenmuseum.

August Kundt erhielt 1870 einen Ruf auf den Lehrstuhl für Physik der Universität Würzburg, und Röntgen konnte ihm als sein sehr geschätzter Assistent dorthin folgen. Doch leider musste er gerade an diesem für ihn später bedeutsamen Ort einen herben Rückschlag in seiner jungen wissenschaftlichen Karriere einstecken. Denn ohne Abitur war eine Habilitation an einer deutschen Universität nicht möglich und trotz wärmster Fürsprache Kundts wurde Röntgen diese auch in Würzburg verwehrt. Ein entscheidender Glücksfall für seine Karriere war der wenig später erfolgende Ruf Kundts an die neugegründete prestigeträchtige „Reichsuniversität“ in Straßburg. Das frisch getraute Ehepaar Röntgen folgte 1872. Die „Reichsuniversität“ war in weiten Teilen progressiv, und aufgrund seiner bisherigen Veröffentlichungen und der Fürsprache seines Mentors Kundts erhielt Röntgen schließlich 1874 die „venia legendi“ und damit das Recht, als Privatdozent an der Universität Vorlesungen zu halten. Ein Ruf als ordentlicher Professor ließ nicht lange auf sich warten, und so ging Röntgen im April 1875 an die Akademie Hohenheim. Die dortigen Verhältnisse führten jedoch dazu, dass er schon im darauffolgenden Jahr als außerordentlicher Professor für Physik an die Universität Straßburg zurückkehrte. 1879 folgte schließlich Röntgens Ruf als ordentlicher Professor der Physik an die Universität Gießen. Zunächst enttäuscht von den dortigen Arbeitsmöglichkeiten, konnte er hier aber nach Schaffung neuer physikalischer Laboratorien, allein und in Zusammenarbeit mit seinen Assistenten, eine Reihe wichtiger Arbeiten auf dem Gebiet der Experimentalphysik leisten. Hier schaffte er sich durch

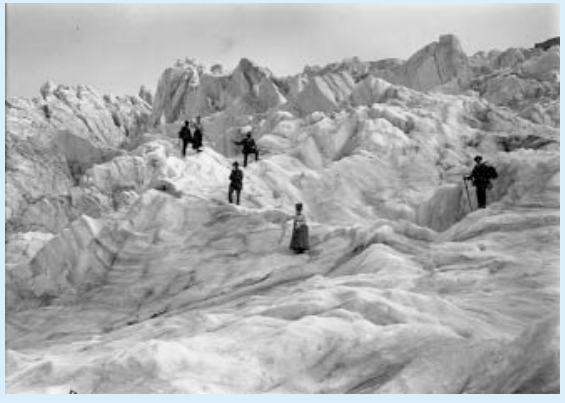

Fotografie Röntgens bei einer Gletscherwanderung. ( ) Deutsches Röntgenmuseum.

seine Veröffentlichungen einen exzellenten wissenschaftlichen Ruf, der schließlich mit einem Angebot einer Professur an der Universität Würzburg und der Leitung des Physikalischen Instituts belohnt wurde. Das fehlende Abitur spielte nun auch keine Rolle mehr.

1888 lässt sich das Ehepaar Röntgen in Würzburg nieder und Röntgen selbst sagt rückblickend über diese Zeit: „Diese zwölf Würzburger Jahre waren meine glücklichsten!“ Das lag unter anderem an dem Institut selbst, das zu einem der modernsten in ganz Deutschland zählte und ihm so exzellente Möglichkeiten für seine Experimente bot, aber sicherlich auch daran, dass er hier seine berühmte Entdeckung der „X-Strahlen“ im Zuge seine Forschung über Kathodenstrahlung machte. Dies geschah am 8. November 1895 und wurde am 10. Dezember 1901 mit dem ersten Nobelpreis überhaupt honoriert. Den in den Statuten geforderten Vortrag bei der Verleihung hat Röntgen aufgrund seines "Lampenfiebers“, wie er schrieb, nie gehalten. Mit seiner Entdeckung in die Weltöffentlichkeit zu geraten gefiel Röntgen, der als eher bescheidener und introvertierter Mensch beschrieben wird, nicht gerade. Für ihn zählten fachliche Reputation und wissenschaftlicher Fortschritt mehr - so beschwert er sich über den Rummel kurz nach seiner Entdeckung wie folgt: „[...], aber Zeit hat der Sturm gekostet, gerade 4 volle Wochen bin ich nicht zu einem Versuch gekommen. Andere Leute konnten arbeiten, nur ich nicht. - Sie haben keinen Begriff davon, wie es hergegangen ist.“

Röntgen war aber bei Weitem kein Mensch, der ausschließlich gearbeitet hat. Im Frühjahr reiste das Ehepaar Röntgen oft für einige Wochen nach Italien, und die Sommerurlaube verbrachte es seit 1873 regelmäßig in Pontresina in der Schweiz, wo sich ein fester Kreis von Freunden jedes Jahr wieder einfand. „Mit vier Wochen Pontresina verlängere ich jeweils mein Leben um ein Jahr“, so fasste Röntgen seine Begeisterung für den auf 1805 m Höhe gelegenen Ort im Engadin zusammen. Dort konnte Röntgen gleich 2 seiner Leidenschaften nachgehen - zum einen dem Fotografieren, ein Hobby, das später auch für seine Entdeckung eine wichtige Rolle gespielt hat, und zum anderen dem Bergsteigen. Letzteres führte ihn auch in die ein oder andere brenzlige Situation, und so resümierte Röntgen in späteren Jahren selbst: „Am liebsten ist es mir noch immer, von den begangenen Wegen abzugehen und über Stock und Stein zu wandern. Ich sagte schon Ritzmann, wenn ich einmal vermisst werden sollte, so sucht mich nicht auf der Landstrasse.“ Dazu ist es zum Glück nicht gekommen, denn seine letzte Lebensstation sollte München werden. 1900 folgte er dem Ruf als ordentlicher Professor für Physik an die Ludwig-Maximilians-Universität. Nicht viel später erwarb er auch sein „Jagdhäusel“ in dem von München aus mit der Eisenbahn leicht zu erreichenden Weilheim. Schon in Gießener und Würzburger Zeit war er ein begeisterter Jäger und zog gerne mit seiner „geliebten Schrotflinte" durch Wald und Flur.

Noch in den letzten Lebenstagen seiner Frau im Jahr 1919 beantragte er die Emeritierung vom Lehrbetrieb, behielt jedoch Direktion über die physikalisch-metronomische Sammlung und das Benutzungsrecht zweier Zimmer am Physikalischen Institut, sodass er weiter die Möglichkeit zu wissenschaftlicher Arbeit hatte. Die Erinnerung an seine Frau und ihre gemeinsame Zeit prägte seine letzten Jahre - er zog sich privat immer mehr zurück und hielt nur noch mit wenigen engen Freunden Kontakt. 1923 verstarb er mit 78 Jahren in seiner Münchener Wohnung.

\section{Literatur}

[1] Glasser O. Wilhelm Conrad Röntgen, Berlin 1931/1958/1995.

[2] Ritzmann K. Wilhelm Conrad Röntgen und die Schweiz, Remscheid 2001. 